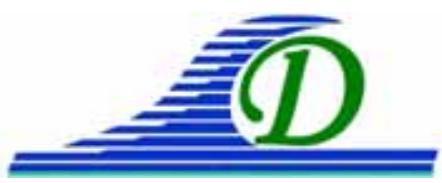

XIII ${ }^{\text {èmes }}$ Journées Nationales Génie Côtier - Génie Civil Dunkerque, 2-4 juillet 2014

DOI:10.5150/jngcgc.2014.010 (C) Editions Paralia CFL

disponible en ligne - http://www.paralia.fr - available online

\title{
Interaction houle-courant : mesure de la relation de dispersion
}

\section{Pierre-Jean FALTOT ${ }^{1}$, Romain BELLANGER ${ }^{1}$, Jean-Marc MOUGENOT ${ }^{1}$, Germain ROUSSEAUX ${ }^{1}$}

\author{
1. Institut Pprime, UPR 3346, CNRS-Université de Poitiers-ISAE ENSMA \\ 11 Boulevard Marie et Pierre Curie - Téléport 2, BP 30179, 86962 Futuroscope \\ Cedex, France. \\ germain.rousseaux@univ-poitiers.fr
}

\section{Résumé :}

Nous présentons des mesures originales de la relation de dispersion des vagues linéaires en présence d'un courant constant uniforme selon la direction verticale et spatialement homogène dans la direction de contre-propagation. Pour cela, nous utilisons un canal hydraulique SANS batteur à houle : en effet, le bruit spontanément généré par l'installation hydraulique permet de déduire la relation de dispersion des modes excités grâce à la détection sub-pixel de l'interface. Celle-ci est illuminée par une nappe laser qui est absorbée par un colorant fluorescent dans le liquide et est simplement observée à travers la paroi latérale transparente du canal. Nous montrons l'évolution de la relation de dispersion en fonction du débit et de la hauteur d'eau dans le canal avec une concordance excellente avec la théorie. Dans certaines conditions, les modes transverses du canal sont aussi observés. Nous concluons sur la généralisation de nos résultats pour un courant variant dans la direction de propagation longitudinale par exemple en immergeant un obstacle de fond.

Mots-clés : Mécanique des fluides, Ecoulement en canal, Relation de dispersion, Interaction houle-courant, Détection d’interface, Ondes de gravité.

\section{Introduction}

La propagation de la houle dans l'océan, que ce soit au large ou près des côtes est influencée par la présence des courants (de marée, dus au vent, induits par les échanges thermiques, etc...) (PEREGRINE, 1976 ; JONSSON, 1990 ; THOMAS \& KLOPMAN, 1997 ; MAISSA et al., 2013). Par exemples, les courants d'arrachement (ou de bahïnes) rétroagissent sur la houle incidente venue du large induisant un transport sédimentaire qui modifie la bathymétrie complexe à l'origine de ces courants (SCOTT et al., 2011 ; GARNIER et al., 2012) ; les ondes scélérates sont essentiellement observées dans les régions où les vagues de vent se propagent sur un contre-courant comme celui des Aiguilles le long de la côte Est de l'Afrique du Sud (LAVRENOV, 1998 ; LAVRENOV 2003). Le courant modifie la longueur d'onde des vagues ainsi que leur amplitude et direction via le phénomène de réfraction analogue à celui associé au changement de 
bathymétrie (PEREGRINE, 1976 ; JONSSON, 1990 ; THOMAS \& KLOPMAN, 1997). De plus, de nouvelles ondes apparaissent par conversion de mode lorsque le courant varie spatialement comme les ondes bleuies à vitesse de phase et vitesse de groupe opposées (PEREGRINE, 1976 ; MAISSA et al., 2013). Les effets dispersifs induisent par ailleurs l'existence de nombreux points de blocage par le courant pour les différents modes générés (MAISSA et al., 2013) ou bien encore du déferlement suite à la focalisation d'un paquet d'onde à la forme judicieusement préparée durant sa propagation (MERKOUNE et al., 2013). Enfin, l'interaction houle-courant fait l'objet de nombreuses modélisations numériques (BENNIS et al., 2010 ; ZHANG et al., 2010 ; DODET et al., 2012 ; TELES et al., 2012) à travers différents algorithmes et couplages de modèles dont certains basés sur l'approximation WKB (le courant varie lentement dans l'espace) et la connaissance de la relation de dispersion.

Dans ce travail, nous présentons nos mesures en laboratoire de la relation de dispersion d'ondes de gravité dont la fréquence se trouve être modifiée par le terme Doppler associé à la vitesse d'un courant. La relation de dispersion $\omega=\mathrm{f}(\mathrm{k})$ des ondes de gravité traduit l'évolution de leur pulsation $\omega$ en fonction de leur nombre d'onde $\mathrm{k}$ (PEREGRINE, 1976 ; MAISSA et al., 2013). En présence d'un écoulement de vitesse U où on néglige la tension de surface ainsi que l'amplitude des ondes par rapport à la hauteur d'eau, celle-ci est donnée par l'équation suivante (1) :

$(\omega-\mathbf{U} \cdot \mathbf{k})^{2}=g k \tanh (k h)$

En particulier, nous souhaitons apporter une preuve expérimentale de l'existence de modes à fréquence relative négative $\omega$-U. $\mathbf{k}<0$ (WEINFURTNER et al., 2011) souvent négligés en océanographie et génie côtier. Les modifications induites par les variations de la hauteur d'eau ainsi que de débit sont étudiées. Par ailleurs, nous testons les effets de largeur de champ ainsi que de durée d'acquisition.

\section{Présentation du dispositif expérimental}

\subsection{Descriptions du canal et de la méthode optique}

Dans un canal de dimensions $\mathrm{L}=680 \mathrm{~cm} \times \mathrm{W}=39 \mathrm{~cm} \times \mathrm{H}=60 \mathrm{~cm}$, nous imposons un écoulement de débit par unité de largeur q (en $\mathrm{m}^{2} / \mathrm{s}$, q=Q/W avec $\mathrm{Q}$ le débit volumique en $\mathrm{m}^{3} / \mathrm{s}$ ) avec une pompe PCM à rotor excentrée à palier "Moineau" et une technologie de vis sans fin à débit constant (figure 1). Nous mesurons le débit avec un débitmètre PROMAG 55S de chez Hendress-Hauser. Une chambre de convergence tridimensionnelle permet de générer un écoulement uniforme selon la direction verticale (hypothèse validée par nos résultats, cf. paragraphe 4.1 et conclusion) avec l'utilisation de grilles en nid d'abeilles pour minimiser la turbulence en entrée du canal.

Nous éclairons l'écoulement avec un LASER Spectra-Physics 2017 à Argon refroidi à l'eau avec une puissance maximale de 2.2 Watts. Grâce à une lentille cylindrique, nous projetons sur la surface de cet écoulement une nappe laser de $2.5 \mathrm{~mm}$ d'épaisseur. 


\section{XIII ${ }^{\text {èmes }}$ Journées Nationales Génie Côtier - Génie Civil \\ Dunkerque, 2-4 juillet 2014}

Celle-ci se situe au milieu du canal (figures 2 et 3). Pour cela, la sortie du LASER est reliée par une fibre optique à la lentille qui illumine une longueur de canal d'environ 3 mètres. La lentille est placée à 2 mètres au-dessus de la surface de l'eau à l'aide d'un mât qui n'est pas solidaire du canal afin d'éviter la transmission de vibrations mécaniques du canal vers la nappe.

Un colorant fluorescent est mélangé à l'eau : la fluorescéine. Celle-ci a la particularité d'avoir un spectre d'absorption correspondant à celui du LASER, et de réémettre selon une longueur d'onde différente.

Pour une faible concentration en fluorescéine, la nappe LASER traverse la colonne d'eau et permet de visualiser les fluctuations de la surface libre sur le sol (figure 2). Ce sont ces fluctuations qui vont permettre de mesurer la relation de dispersion des vagues.

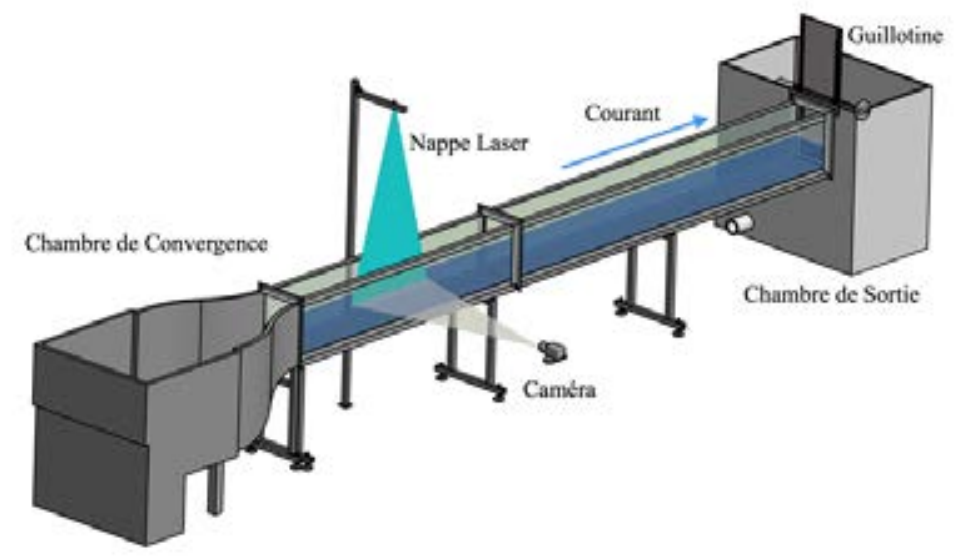

Figure 1. Schéma du dispositif expérimental.

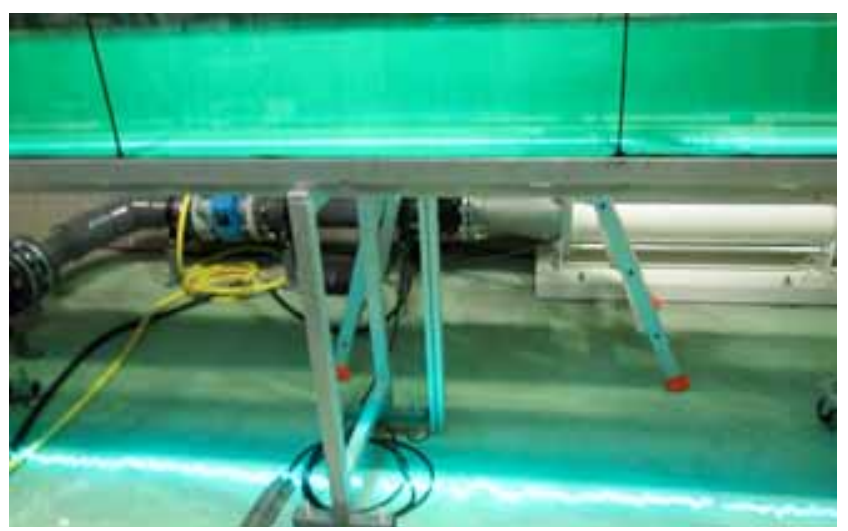

Figure 2. Ecoulement éclairé par la nappe laser avec une faible concentration en fluorescéine dans le fluide.

Une ligne lumineuse apparaît à la surface de l'écoulement pour une forte concentration en fluorescéine (50g pour $24 \mathrm{~cm}$ de hauteur d'eau). En effet, la nappe LASER ne 


\section{Thème 1 - Hydrodynamique côtière}

pénètre que sur une très faible profondeur (quelques $\mathrm{mm}$ ) correspondant à la loi de Beer-Lambert (figure 3).

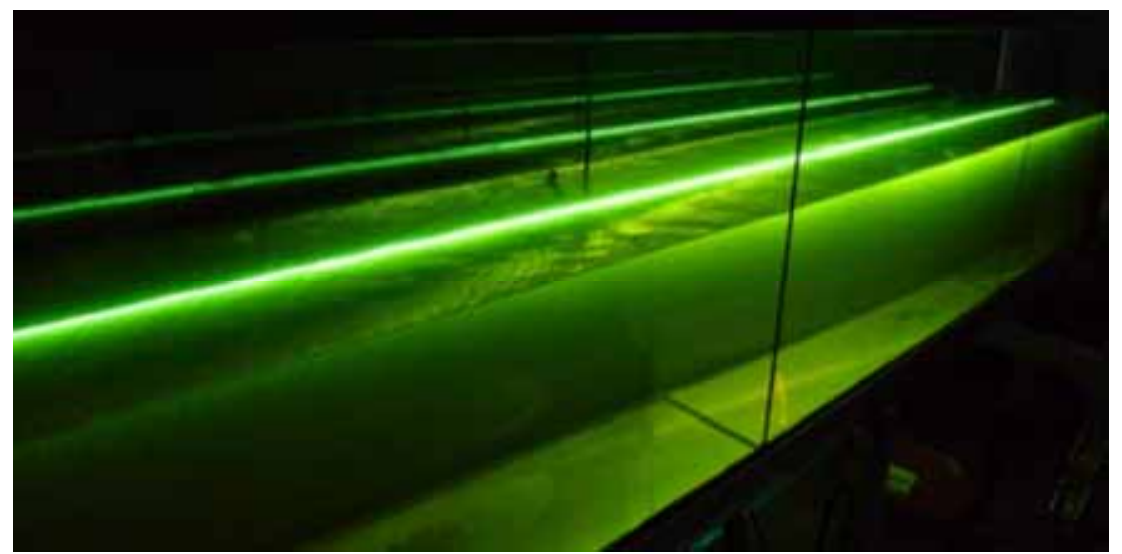

Figure 3. Ecoulement éclairé par la nappe laser avec une forte concentration en fluorescéine dans le fluide.

\subsection{Dispositif d'acquisition}

A l'aide de trois caméras JAI (résolution $1600 \times 1200$ ) disposées côte à côte et à une distance de $2.4 \mathrm{~m}$ d'une des deux parois latérales du canal, nous filmons à 20 images par seconde l'écoulement de côté sur une largeur de $2.80 \mathrm{~m}$. Les caméras font un léger angle avec l'horizontal (environ 7-8 ${ }^{\circ}$ ), de manière à filmer légèrement par-dessus la nappe laser représentant l'interface eau-air afin de ne pas confondre cette interface au milieu du canal avec sa trace due à la diffusion de la lumière sur la paroi latérale. Les trois caméras sont configurées de manière à faire des acquisitions en simultané, chacune filmant une portion bien déterminée du canal d'environ 1 mètre : la reconstruction d'une unique image sous le Logiciel DAVIS de LAVISION nécessite le chevauchement d'environ 10 à 15\% du champ de vision de deux caméras consécutives d'où un champ de vision totale de $2.80 \mathrm{~m}$. La hauteur d'eau en un point est par ailleurs déterminée grâce à des capteurs acoustiques (précision de $0.1 \mathrm{~mm}$ ) placés à la verticale de l'écoulement pour comparaison et étalonnage. Deux raisons principales nous ont menés à utiliser trois caméras : augmenter la largeur de champ pour mesurer des nombres d'ondes petits (cf. discussion ci-dessous sur la résolution spectrale); contourner les montants verticaux noirs correspondants aux raccords entre les plaques de verre constituant les parois du canal (figure 3).

\section{Acquisitions et extraction de l’interface eau-air}

\subsection{Acquisition des images}

Pour chaque acquisition pendant 120 s, nous obtenons une série de 2400 images par caméra. On observe sur la figure 4 l'effet de la parallaxe due à l'angle d'observation. 


\section{XIII ${ }^{\text {èmes }}$ Journées Nationales Génie Côtier - Génie Civil \\ Dunkerque, 2-4 juillet 2014}

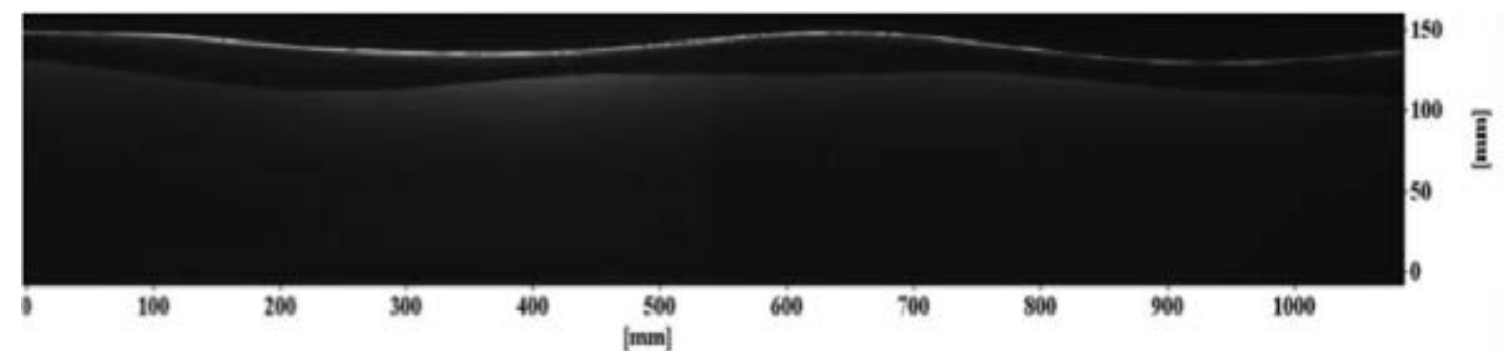

Figure 4. Exemple d'image enregistrée par une caméra. On observe la trace laser au milieu du canal et on devine l'interface sur la paroi latérale due à la diffusion.

Une fois les trois images correspondantes à un instant donné calibrées (prenant compte de la parallaxe grâce à une mire 2D) et assemblées, on obtient une vision de l'écoulement à cet instant le long de la largeur couverte par les trois caméras. L'ensemble des images ainsi assemblées nous fournit alors une vue précise de l'évolution de la surface au niveau de la ligne laser pendant la durée d'acquisition correspondante. La surface libre est déformée à cause de la présence d'ondes de gravité générées par le bruit mécanique du dispositif expérimental, auquel la pompe générant l'écoulement contribue majoritairement.

\subsection{Extraction de l'interface à partir des images}

Sous Matlab, un programme est réalisé permettant de déterminer pour chaque image la position de l'interface avec une précision subpixel (typiquement au centième de pixel). Si on trace l'évolution de l'intensité le long d'une ligne verticale de pixels sur l'image, on constate en effet un pic de forme très proche d'une gaussienne. On interpole donc ce profil d'intensité avec une fonction gaussienne selon la verticale. Sur la courbe ainsi obtenue, on place alors l'interface au niveau du maximum. En réalisant cette interpolation pour chaque colonne de l'image, on arrive ainsi à placer très précisément point par point l'interface eau-air (cf. figure 5).

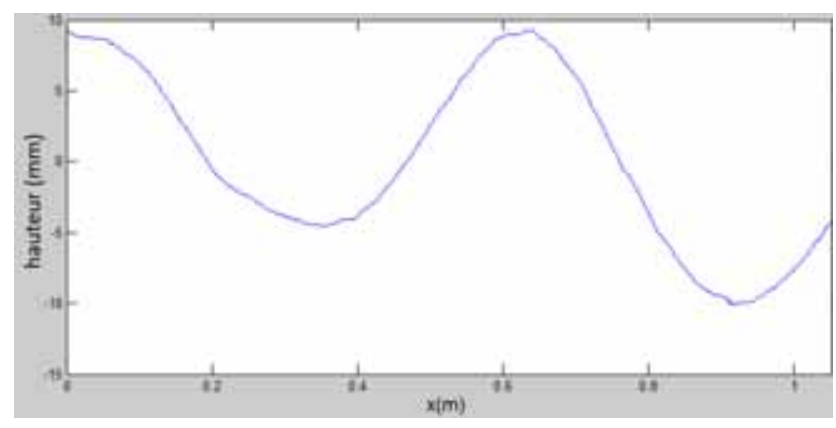

Figure 5. Interface extraite correspondante à la figure 4 avec une détection sub-pixel. 


\section{Thème 1 - Hydrodynamique côtière}

On constate la présence d'une ondulation à grande échelle (amplitude d'environ 20mm) sur laquelle se superposent les fluctuations. Cette ondulation est la solution de fréquence nulle de la relation de dispersion, encore appelée mode zéro qui est de nombre d'onde non-nul du fait de la présence d'une vitesse non-nulle (MAISSA et al., 2013).

\section{Tracé expérimental de la relation de dispersion}

\subsection{Calcul et affichage de la relation de dispersion}

Les 2400 interfaces extraites pour une acquisition nous donnent une évolution dans le temps (mouvement d'un point d'une image à l'autre) et dans l'espace (variations de hauteur sur une image). On en déduit le diagramme spatio-temporel de la figure 6. On note la présence de fluctuations temporelles importantes. De plus le niveau moyen baisse de la gauche vers la droite c'est-à-dire dans le sens de l'écoulement à cause des pertes de charges et du mode zéro (MAISSA et al., 2013).

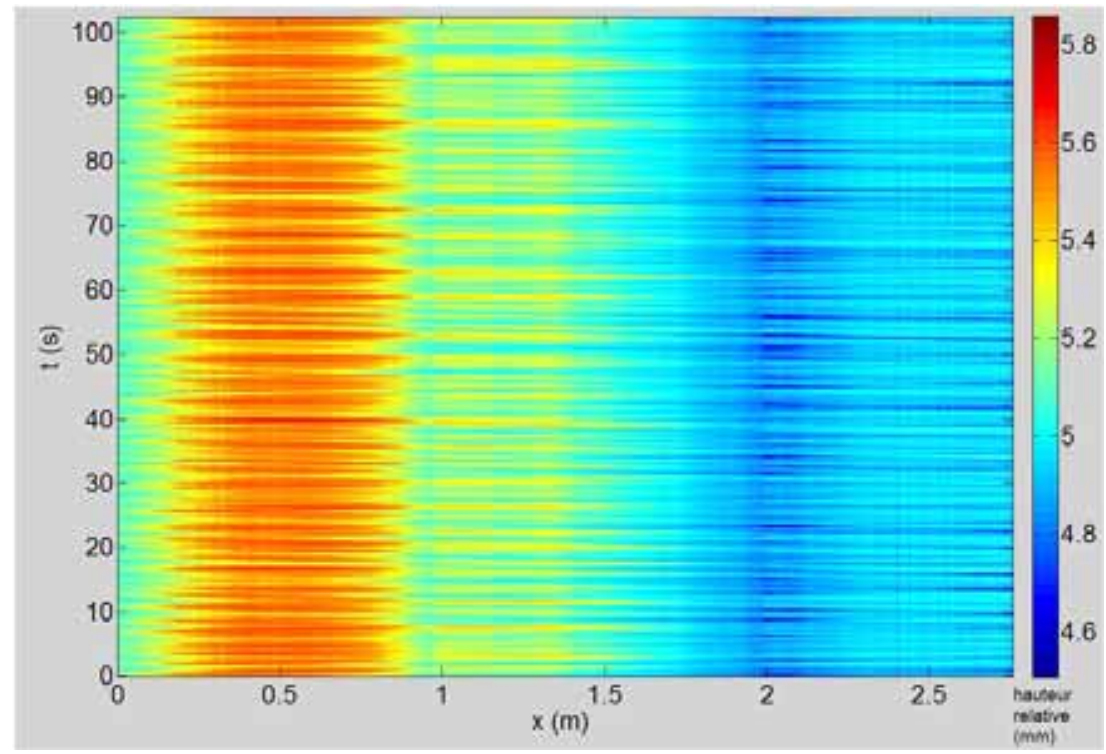

Figure 6. Diagramme spatio-temporel. La hauteur d'eau statique est $24 \mathrm{~cm}$ et le débit est $q=0.038 \mathrm{~m}^{2} / \mathrm{s}$. La hauteur relative par rapport au repos est indiquée dans le code des couleurs à droite.

On peut résumer ces évolutions dans une matrice où une ligne représente les évolutions spatiales à un instant donné, et une colonne les évolutions temporelles à une position donnée. Une double transformée de Fourier dans l'espace et dans le temps (FFT 2D) est alors réalisée sur cette matrice afin de déterminer tous les modes qui composent l’interface. On obtient la relation de dispersion présentée en figure 7. 


\section{XIII ${ }^{\text {èmes }}$ Journées Nationales Génie Côtier - Génie Civil \\ Dunkerque, 2-4 juillet 2014}

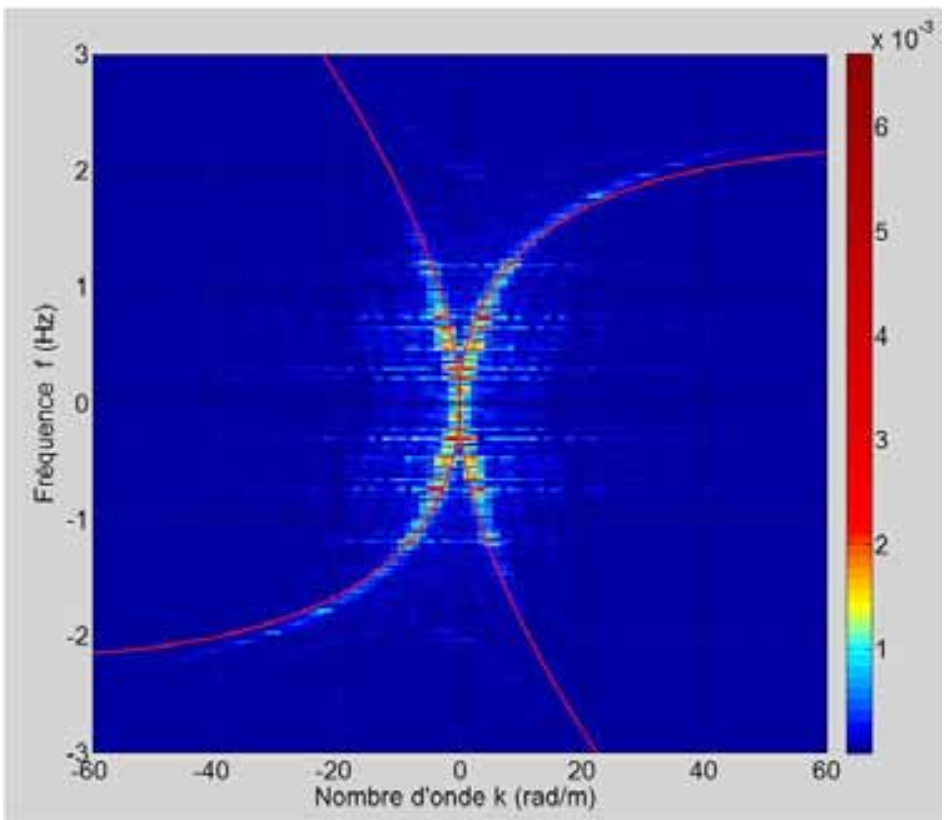

Figure 7. Relation de dispersion d'un écoulement de hauteur d'eau statique $24 \mathrm{~cm}$ et de débit $q=0.038 \mathrm{~m}^{2} / \mathrm{s}$ (en rouge : la courbe théorique de la relation de dispersion).

On observe que de très nombreux modes sont générés par le bruit ambiant, et que la réunion de tous les points correspondants constitue un tracé expérimental très précis de la relation de dispersion dans une gamme de nombres d'onde allant de -50 à $50 \mathrm{~m}^{-1}$. De plus, lorsque l'on superpose à ce tracé la courbe théorique donnée selon l'équation (1), on observe une excellente correspondance entre les deux.

Afin d'arriver à ce résultat, nous avons dû optimiser un certain nombre de réglages. En effet, notons $\Delta k_{x}$ la largeur d'un échantillon de la FFT spatiale (figure 8) :

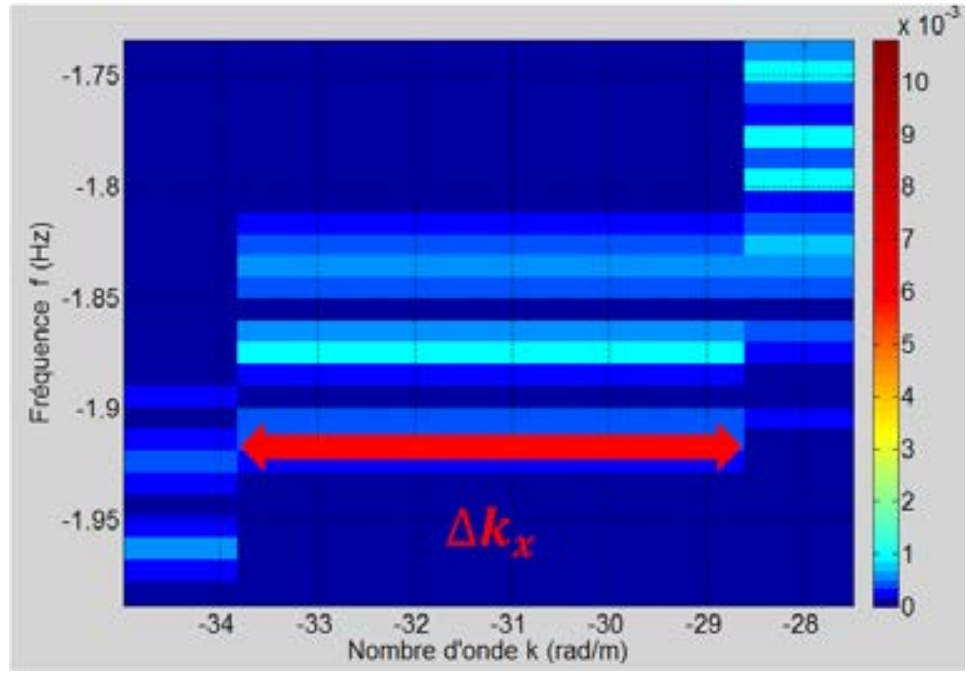

Figure 8. Zoom sur la relation de dispersion : on observe la taille de l'échantillonnage spatial. 


\section{Thème 1 - Hydrodynamique côtière}

La fréquence spatiale d'échantillonnage est $k_{s}=2 \pi / d x$, où $\mathrm{dx}$ représente la taille en mètres d'un pixel sur l'image. Nous pouvons donc observer sur le résultat de la FFT des nombres d'onde compris entre $-k_{s} / 2$ et $k_{s} / 2$ après centrage. De plus, le signal spatial comportant Nx points (largeur de l'image en pixels), la FFT comportera également Nx points horizontalement (figure 9) :

On en déduit que :

$$
\Delta k_{x}=\frac{k_{s}}{N_{X}}=\frac{2 \pi}{N_{X} d x}
$$

Or $N_{x} d x=L$ est la largeur réelle de canal observée.

Donc, plus le champ de vision est large (quel que soit la résolution spatiale du capteur), meilleure sera la résolution spectrale et plus précise sera la FFT.

Avec une largeur de champ de $2.8 \mathrm{~m}$, nous accédons à une résolution spectrale en de l’ordre de $2.24 \mathrm{rad} / \mathrm{m}$ sachant que la résolution spatiale avec les 3 caméras est de 0.64 $\mathrm{mm} /$ pixel.

Plusieurs méthodes d'interpolation sub-pixel ont été testées comme l'interpolation par un polynôme du second degré ou cubique, l'interpolation avec une gaussienne donne les meilleurs résultats car proche de la forme de l'intensité autour du maximum de la nappe LASER.

Remarque : Pour faciliter l'observation et pour la mise en page dans la suite de l'article, nous avons supprimé les échelles dans la relation de dispersion. La gamme de variation de nombres d'ondes est toujours entre -60 et $+60 \mathrm{~m}^{-1}$; de même, la gamme en fréquence est toujours entre -3 et $+3 \mathrm{~Hz}$ comme pour la figure 7 .

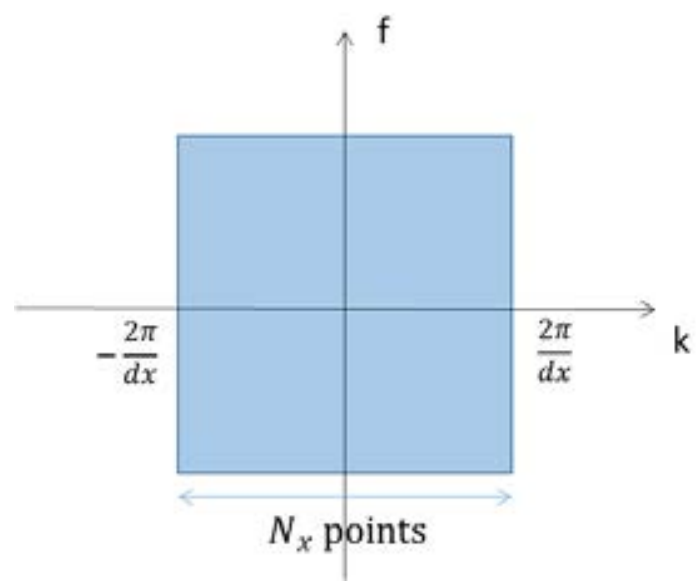

Figure 9. Caractéristiques de la FFT 2D, le carré bleu représente l'étendue du résultat.

\subsection{Influence du nombre de points}

On rappelle que le nombre de points est proportionnel à la largeur de champ observé. Pour chaque image, l'interface est représentée par un tableau dont chaque élément représente la hauteur du pixel associé. Nous réduisons la taille de ce tableau en enlevant des points de façon homogène (figure 10 (a), (b) et (c)). 


\section{XIII ${ }^{\text {èmes }}$ Journées Nationales Génie Côtier - Génie Civil \\ Dunkerque, 2-4 juillet 2014}

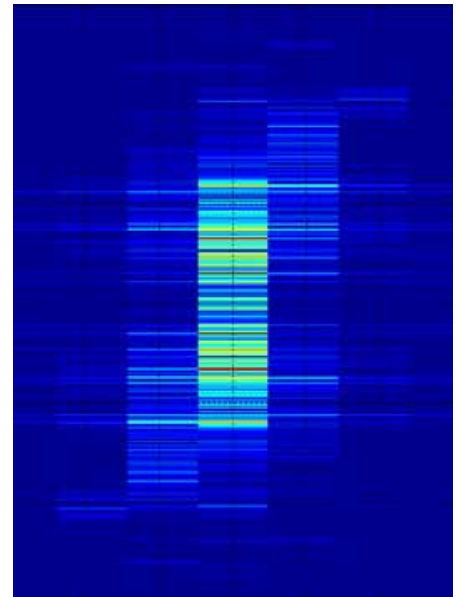

Figure 10 (a). Relation de dispersion à $h=24 \mathrm{~cm}$, $q=0.038 \mathrm{~m}^{2} / \mathrm{s}, 512$ points.

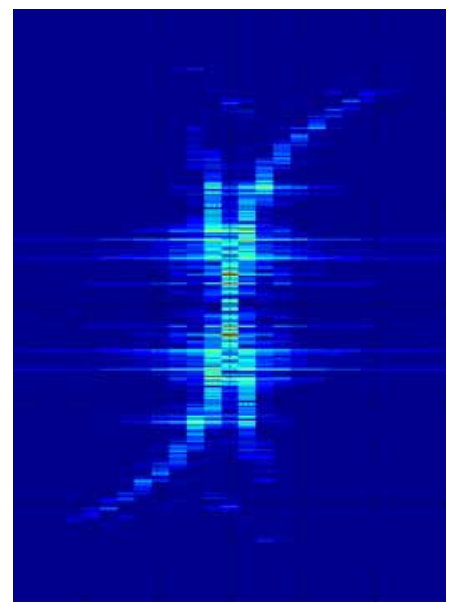

Figure 10 (b). Relation de dispersion à $h=24 \mathrm{~cm}$, $q=0.038 \mathrm{~m}^{2} / \mathrm{s}, 2048$ points.

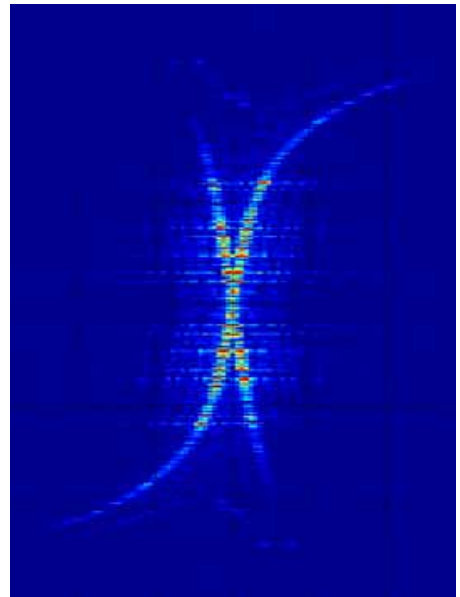

Figure 10 (c). Relation de dispersion à $h=24 \mathrm{~cm}$, $q=0.038 \mathrm{~m}^{2} / \mathrm{s}, 8192$ points.

Cette fois ci, c'est logiquement sur la résolution horizontale que l'on joue. Modifier simultanément le nombre d'images et le nombre de points revient donc à modifier les dimensions des rectangles qui sont les plus petits échantillons de cette FFT 2D.

Le problème est analogue pour la résolution dans le sens des fréquences qui est proportionnelle à la durée de l'acquisition.

\subsection{Influence du nombre d'images}

Diminuer le nombre d'images revient à diminuer la résolution verticale (dans le sens des fréquences). Cependant, avoir une FFT 2D avec des échantillons carrés peut faciliter son étude.

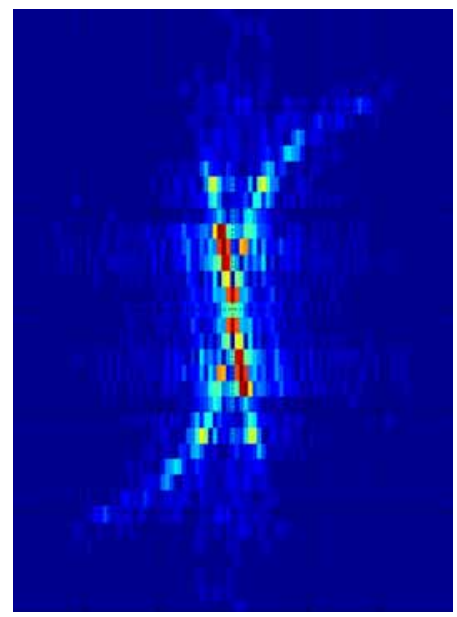

Figure 11 (a). Relation de dispersion à $h=24 \mathrm{~cm}$, $q=0.038 \mathrm{~m}^{2} / \mathrm{s}, 128$ images.

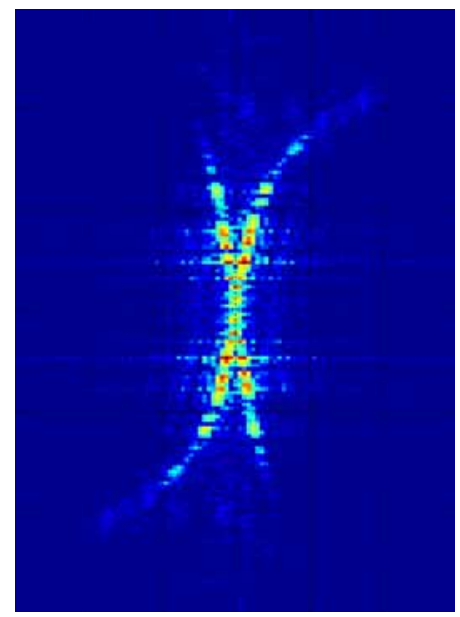

Figure 11 (b). Relation de dispersion à $h=24 \mathrm{~cm}$, $q=0.038 \mathrm{~m}^{2} / \mathrm{s}, 512$ images.

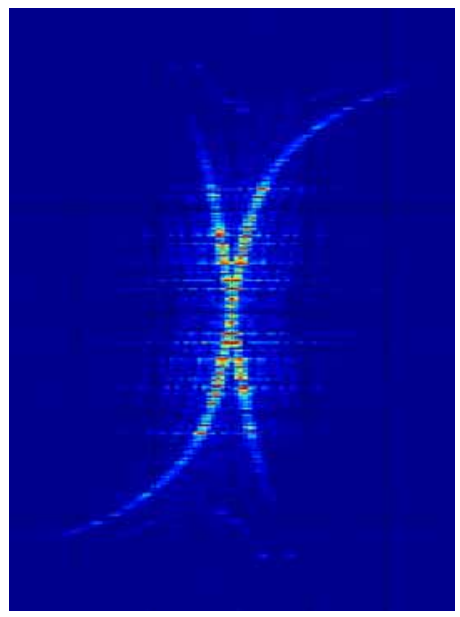

Figure 11 (c). Relation de dispersion à $h=24 \mathrm{~cm}$, $q=0.038 \mathrm{~m}^{2} / \mathrm{s}, 2048$ images. 


\section{Thème 1 - Hydrodynamique côtière}

\subsection{Influence du débit à hauteur statique fixée}

On observe plusieurs effets sur les relations de dispersion en augmentant le débit (figure 12) : les branches dispersives tournent en fonction de la vitesse comme attendu théoriquement (MAISSA et al., 2013); on observe bien les deux branches correspondant aux deux signes plus et moins dans la relation de dispersion. En effet, la relation de dispersion peut s'écrire de manière équivalente à l'équation (1) :

$\omega=\mathbf{U} \cdot \mathbf{k} \pm \sqrt{g k \tanh (k h)}$

Cette forme démontre de manière indiscutable l'existence d'une branche négative en plus de la branche positive souvent et uniquement envisagée dans la littérature. On trace cette relation de dispersion théoriquement sur la figure 13 pour différentes vitesses pour comparaison. La branche négative en bleu de la figure 13 génère une solution supplémentaire à fréquence positive dans le référentiel du labo mais à fréquence relative négative dans le référentiel du courant (MAISSA et al., 2013) ce qui peut se voir en écrivant la relation de dispersion sous cette troisième forme équivalente (3) :

$\omega-\mathbf{U} \cdot \mathbf{k}= \pm \sqrt{g k \tanh (k h)}$

Cette solution supplémentaire n’aurait pas été identifiée sans la prise en compte du signe négatif dans les équations (2) ou (3).

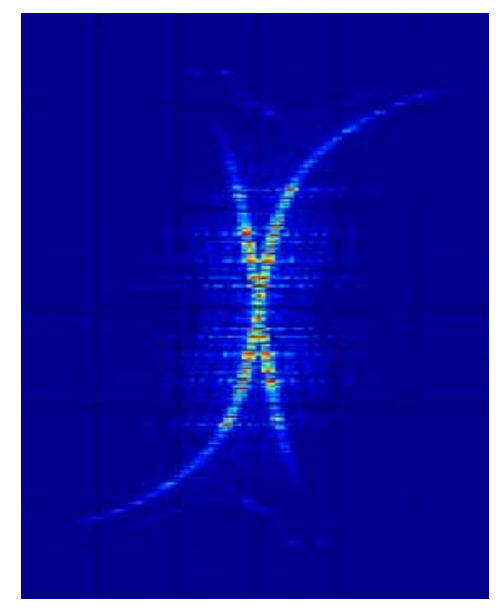

Figure 12 (a). Relation de dispersion à $h=24 \mathrm{~cm}$, $q=0.038 \mathrm{~m}^{2} / \mathrm{s}$.

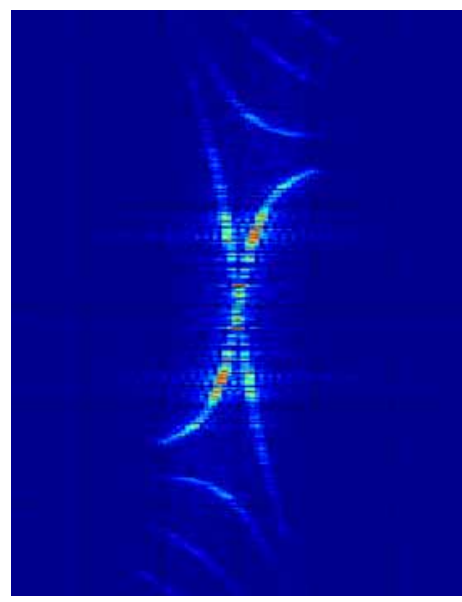

Figure 12 (b). Relation de dispersion à $h=24 \mathrm{~cm}$, $q=0.064 \mathrm{~m}^{2} / \mathrm{s}$.

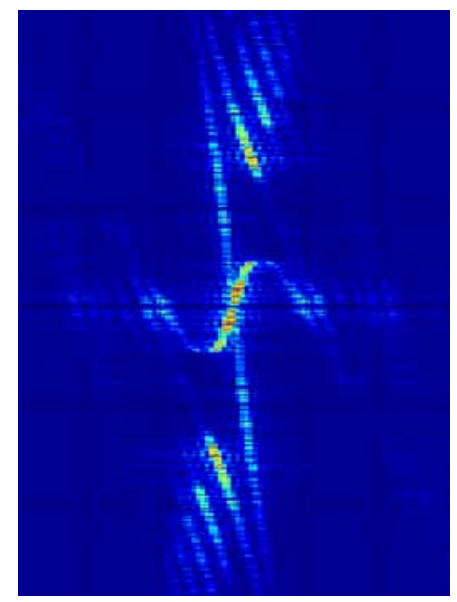

Figure 12 (c). Relation de dispersion à $h=24 \mathrm{~cm}$, $q=0.16 \mathrm{~m}^{2} / \mathrm{s}$.

Concernant nos mesures expérimentales, le résultat à plus haut débit (figure 12 (c)) montre que, même pour une fréquence fixée positive dans le référentiel du laboratoire, quatre solutions sont possibles et pas simplement deux à petit débit (figure 12 (a) et (b)). 


\section{XIII ${ }^{\text {èmes }}$ Journées Nationales Génie Côtier - Génie Civil \\ Dunkerque, 2-4 juillet 2014}

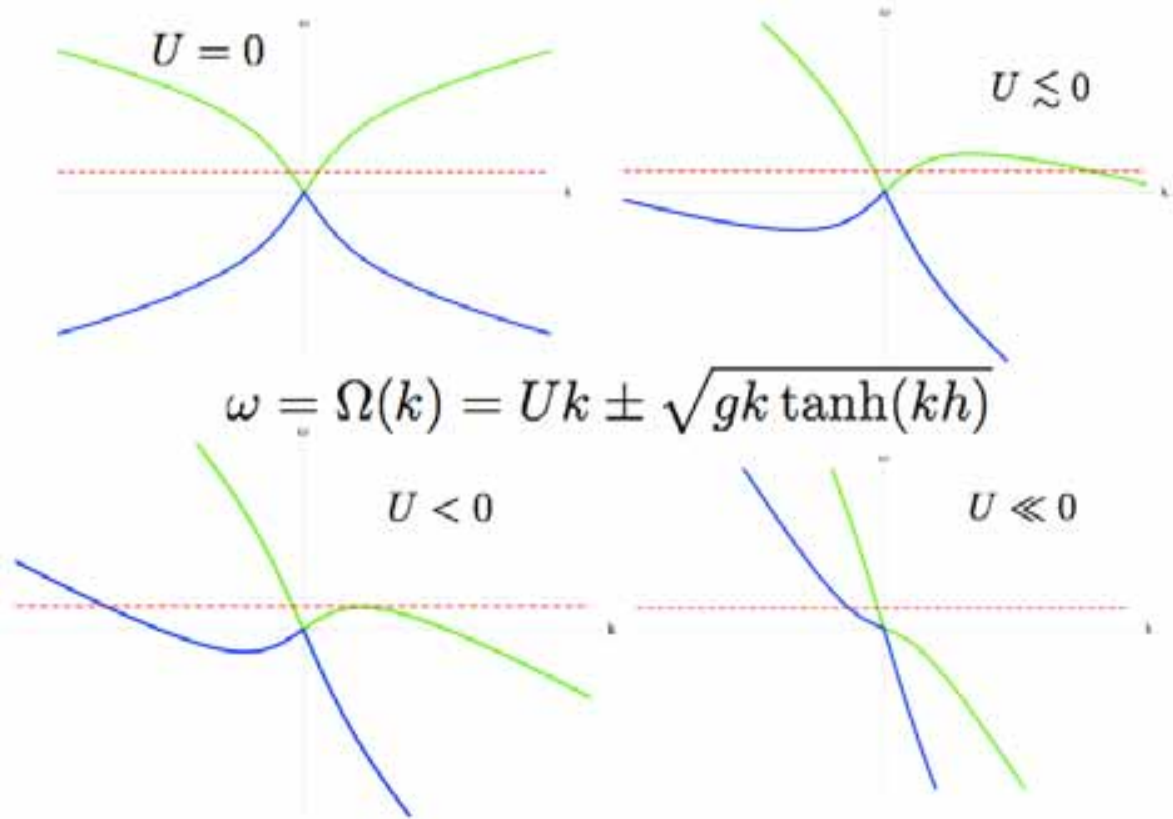

Figure 13. Relation de dispersion théorique avec ses branches positive (en vert) et négative (en bleu) pour différentes vitesses du contre-courant.

De plus, on observe des modes transverses particulièrement quand le bruit est important (figure 12 (c)) qui sont théoriquement confirmés en se rappelant que la nappe LASER mesure le nombre d'onde longitudinal et que le nombre d'onde non projeté s'écrit $k=\left(k_{x}^{2}+k_{y}^{2}\right)^{1 / 2}$ avec $k_{y}=n . \pi / W$ où $\mathrm{n}$ est un entier indexant les modes et $\mathrm{W}$ est la largeur du canal. Le mode transverse est quantifié par annulation de la vitesse normale sur les deux parois latérales.

\subsection{Influence de la hauteur d'eau statique à un débit fixé}

De manière similaire à l'augmentation de débit, la diminution de niveau d'eau amplifie le bruit dans l'installation d'où une meilleure observation de la relation de dispersion à faible hauteur initiale (figure 14 (a) et (b)). Pour les plus grandes hauteurs (figure 14 (c)), seul le comportement en ondes longues est observé pour les nombres d'ondes proches de zéro. 


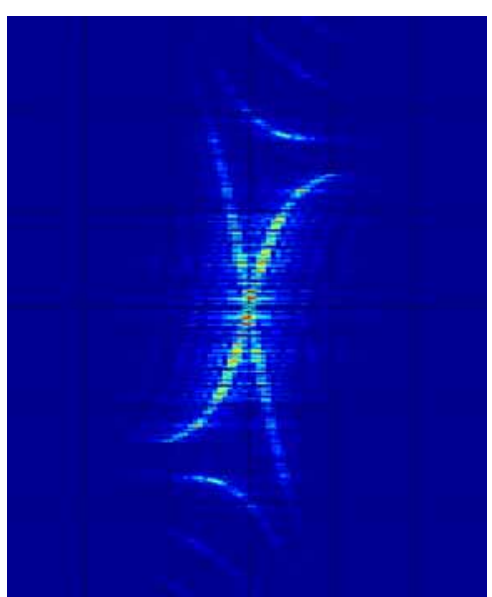

Figure 14 (a). Relation de dispersion à $h=15 \mathrm{~cm}$, $q=0.038 \mathrm{~m}^{2} / \mathrm{s}$

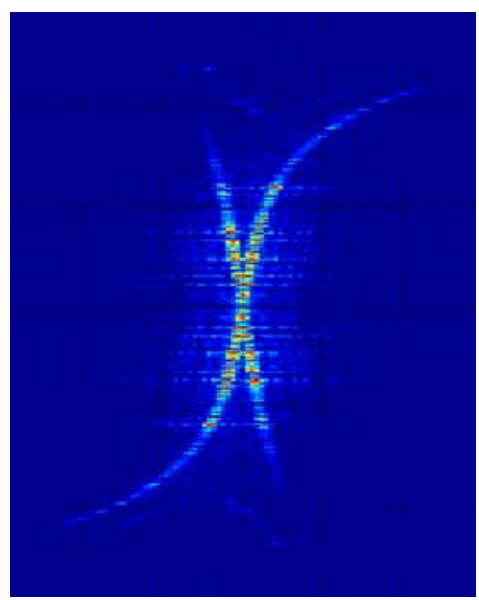

Figure 14 (b). Relation de dispersion à $h=24 \mathrm{~cm}$, $q=0.038 \mathrm{~m}^{2} / \mathrm{s}$.

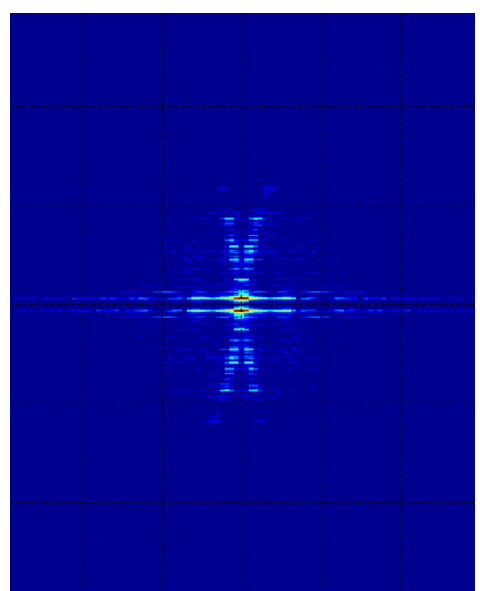

Figure 14 (c). Relation de dispersion à $h=35 \mathrm{~cm}$, $q=0.038 \mathrm{~m}^{2} / \mathrm{s}$.

\section{Conclusions et perspectives}

Nous avons mesuré la relation de dispersion des vagues de gravité linéaires constituant la houle en présence d'un courant en exploitant les fluctuations de la surface libre d'origine mécanique spontanément présentes en raison des bruits de notre canal hydraulique. Ainsi, nous évitons de manière astucieuse une étude fastidieuse avec un batteur à houle classique qui obligerait l'expérimentateur à mesurer la longueur d'onde de l'onde incidente en fonction de la fréquence du batteur. La tâche est d'autant plus compliquée que de nouveaux modes (bleuis et négatifs) sont générés par l'interaction avec l'onde incidente (MAISSA et al., 2013) et il est d'autant plus difficile de séparer les modes et de tracer la relation de dispersion "pas à pas" c.a.d. fréquence par fréquence. Tout se passe comme si le bruit mécanique jouait le rôle d’une assemblée de batteurs à des fréquences différentes qui viennent exciter simultanément la surface libre. Si le débit diminue ou si la hauteur d'eau augmente, le bruit diminue et il est plus difficile d'exhiber la relation de dispersion qui se construit par l'accumulation statistique de l'amplitude du bruit sur les branches dispersives.

Du point de vue des applications concrètes pour le génie côtier, les modes supplémentaires générés par l'interaction entre une houle incidente et un courant (en particulier ceux à fréquences relatives négatives) doivent être pris en compte dans la prédiction de la surface libre par les codes numériques, dans les modélisations et par les expérimentateurs. Nous avons considéré que la houle pouvait être décrite par une relation de dispersion : il resterait à prendre en compte dans une future étude les effets de la non-linéarité en amplitude, de la tension de surface et de la vorticité sur la relation de dispersion dans le cas d'une houle naturelle (MAISSA et al., 2013).

Nous avons fait l'hypothèse que la vitesse de l'écoulement ne variait pas dans la direction longitudinale ce qui est vrai à condition de négliger les pertes de charges sur le 


\section{XIII ${ }^{\text {èmes }}$ Journées Nationales Génie Côtier - Génie Civil \\ Dunkerque, 2-4 juillet 2014}

champ de vision des caméras. Si l'on insère un obstacle pour rendre inhomogène le profil de vitesse longitudinale, tout se passe comme si la relation de dispersion était la superposition des relations de dispersion obtenues pour une vitesse donnée dans la gamme de variation de vitesse induite par l'obstacle. Nous sommes en train d'étudier la généralisation du concept de relation de dispersion en présence d'un milieu inhomogène. La notion même de diagramme spatio-temporelle doit être généralisée pour tenir compte de l’inhomogénéité...

Nous étudierons par ailleurs l'influence sur la relation de dispersion d'un profil de vitesse non-uniforme selon la verticale (MAISSA et al., 2013) comme nous en avons fait l'hypothèse dans ce travail : pour cela, la chambre de convergence peut devenir 2D et l'on peut modifier la nature du fond du canal en rajoutant des rugosités effectives. L'étalonnage hydraulique du canal (non reporté dans ce travail) démontre la véracité de cette hypothèse. Par manque de place, nous n'avons pas incorporé l'étude des champs de vitesse dans cet article que nous avons effectué avec un ADV et par PIV. Il suffit de constater que les courbes rouges théoriques sont en bon accord avec nos résultats expérimentaux pour valider ce fait car dans le cas contraire il y aurait des écarts dus à la modification de la relation de dispersion par la présence de vorticité (MAISSA et al., 2013).

\section{Remerciements :}

G.R. souhaite remercier vivement les organismes suivants pour leur soutien et les subventions accordées: la Mission Interdisciplinaire du CNRS, l'ACI Interaction Houle-Courant de l'Université de Poitiers 2013 ainsi que le FEDER 35790-2012 qui a financé le canal.

\section{Références bibliographiques}

BENNIS A.-C., ARDHUIN F., ODAKA T. (2010). Modélisation couplée vaguescourant : développements avec MARS3D et WAVEWATCH III. Journées Nationales Génie Côtier - Génie Civil, Sables d’Olonne, pp 17-24. http://dx.doi.org/10.5150/jngcgc.2010.003-B

DODET G., BERTIN X., BRUNEAU N., FORTUNATO A., NAHON A., TABORDA R. (2012). Interactions vagues-courants dans une embouchure tidale dominée par la houle. Journées Nationales Génie Côtier - Génie Civil, Cherbourg, pp 41-50. http://dx.doi.org/10.5150/jngcgc.2012.005-D

GARNIER R., FALQUÉS A., DODD N. (2012). Origine des courants d'arrachement bien établis. Journées Nationales Génie Côtier - Génie Civil, Cherbourg, pp 67-80. http://dx.doi.org/10.5150/jngcgc.2012.008-G

JONSSON I.G. (1990). Wave-current interactions. In The Sea (ed. B. Le Mehaute and D. M. Hanes), pp 65-120, John Wiley. 
LAVRENOV I.V. (1998) The wave energy concentration at the Agulhas current off South Africa. Natural Hazards, Vol. 17, pp 117-127. http://dx.doi.org/10.1023/A:1007978326982 LAVRENOV I.V. (2003). Wind-Waves in Oceans, Springer. http://dx.doi.org/10.1007/978-3$\underline{662-05146-7}$

MAISSA P., ROUSSEAUX G., STEPANYANTS Y. (2013). Influence of shear-flow vorticity on wave-current interaction. 7th International Conference on Coastal Dynamics, Arcachon, pp 1137-1146. http://www.coastaldynamics2013.fr/pdf files/103 Maissa Philippe.pdf MERKOUNE D., TOUBOUL J., ABCHA N., MOUAZE D., EZERSKY A. (2012). Interaction houle focalisée-courant dans une profondeur finie. Journées Nationales Génie Côtier - Génie Civil, Cherbourg, pp 101-108. http://dx.doi.org/10.5150/jngcgc.2012.011-M PEREGRINE D.H. (1976). Interaction of water waves and currents. Adv. Appl. Mech., Vol. 16, pp 9-117. http://dx.doi.org/10.1016/S0065-2156(08)70087-5

SCOTT T., MASSELINK G., RUSSELL P., AUSTIN M., WILLS S., WOOLER A. (2011). Rip current hazards on large-tidal beaches in the UK. In: Leatherman, S., Fletemeyer, J. (Eds.), Rip Currents: Beach Safety, Physical Oceanography, and Wave Modeling. CRC Press, pp 225-243. http://dx.doi.org/10.1201/b10916-15

TELES M.J., BENOIT M., PIRES-SILVA A.A. (2012). Analysis of vertical profiles of mean velocity, shear stress and turbulent viscosity in a wave-current environment through RANS numerical simulations. Journées Nationales Génie Côtier - Génie Civil, Cherbourg, pp 133-140. http://dx.doi.org/10.5150/jngcgc.2012.015-T

THOMAS G.P., KLOPMAN G. (1997). Wave-current interactions in the nearshore region. In Gravity Waves in Water of Finite Depth. J. N. Hunt, Editor, Advances in Fluid Mechanics, Vol. 10, CMP, Southampton.

WEINFURTNER S., TEDFORD E.W., PENRICE M.C.J., UNRUH W.G., LAWRENCE G.A. (2011). Measurement of stimulated Hawking emission in an analogue system. Physical Review Letters, Vol. 106, 021302. http://dx.doi.org/10.1103/PhysRevLett.106.021302

ZHANG B., DEBAILLON P., SERGENT P. (2010). Modélisation numérique du couplage houle - courants pour l'agitation de houle. Comparaison avec des essais en cuve à houle, Journées Nationales Génie Côtier - Génie Civil, Sables d’Olonne, pp 147154. http://dx.doi.org/10.5150/jngcgc.2010.018-Z 